\title{
AN INVESTIGATION OF THE MYOPIA FOR FUTURE CONSEQUENCES THEORY OF VMF PATIENT BEHAVIOUR ON THE IOWA GAMBLING TASK; AN ABSTRACT NEURAL NETWORK SIMULATION
}

\author{
KIRAN KALIDINDI, HOWARD BOWMAN AND BRADLEY WYBLE \\ Centre for Cognitive Neuroscience and Cognitive Systems, Computing Laboratory, \\ University of Kent, Canterbury, Kent CT2 7NF, England. \\ (kk49@kent.ac.uk, H.Bowman@kent.ac.uk, and B.Wyble@kent.ac.uk)
}

\begin{abstract}
'Somatic marker' theory proposes that body states act as a valence associated with potential choices based on prior outcomes; and thus aid decision-making. The main supporting evidence for this theory arose from clinical interviews of subjects with ventromedial prefrontal cortex (VMF) lesions and their performance on the Iowa 'Gambling Task' (IGT). VMF patient behaviour has been described as 'myopia' about future consequences. The aim of this paper is to investigate the implications of this description using an abstract simulation of the neural mechanisms that could underlie decision-making in this type of reinforcement learning task.
\end{abstract}

\section{Introduction}

Over the past decade there has been an increasing interest in the role of emotions in everyday decision-making. A theory particularly well represented in the literature is A. Damasio's 'somatic marker' theory (Damasio, 1994), which proposes that body states act as a valence which can be associated with potential choices based on prior outcomes; and thus aid decision-making. The main supporting evidence for this theory arose from clinical interviews of subjects with ventromedial prefrontal cortex (VMF) lesions and their performance on the Iowa 'Gambling Task' (IGT) (Bechara, 1999), compared to normal controls and those with lesions in other brain areas. The IGT consists of four decks that subjects can pick from; two decks, A and B, which yield high wins but higher losses (Disadvantageous) and the other two, C and D, that yield low wins with lower losses (Advantageous). Normal subjects start by picking from the disadvantageous decks but learn to pick from the advantageous decks, unlike the VMF patients who, as in their real social and personal lives, continue to pick disadvantageously.

A description that accounts for this behaviour in VMF patients' is 'myopia' for future consequences, in that they are driven by immediate reward and are less interested in uncertain future loss or gain (Bechara et al, 2000a). The main aim of this paper is to investigate the implications of this 'myopia' with an abstract neural network simulation that could characterize decisionmaking in this type of task. The current literature lacks a model that accurately 
reproduces these experimental results, and is abstracted from specific anatomical details, which underlie other models e.g. Wagar and Thagard (2004).

This model focuses on a time averaging parameter $\tau$, which dictates the relative influence of current and past information in decision-making. A 'myopia' for future consequences could be caused by a similar 'myopia' for past events, where the experience from those events is not well integrated into current decision-making. Exploration of the state space for $\tau$, from constant values to linear or logarithmic growth over the 100 trials in each example of the task, found that certain values of $\tau$ provided a good fit to the experimental data for normal controls and VMF subjects. This investigation informs us about how these different groups may utilise past information in present decision-making, especially in relation to situational novelty.

\section{Design}

In the design of the neural network, attempts were made to make the least number of assumptions and still produce an abstract network that can replicate the human data. The model makes the following basic assumptions:

1. That pleasure and pain are equally valued by Normal controls and VMF patients of the IGT.

2. An activation-based memory is sufficient to hold the required information about the task.

Based on the above assumptions, the network shown in Figure 1 was designed and implemented as a possible solution.

For each choice, there are two memory units which represent a measure of expected value, with one unit recording positive information (the positive unit) resulting from a choice and the other unit negative information (the negative unit). The results of a particular choice are passed on to the relevant Memory Layer units by having the unit associated with the current choice active in the Result Layer, while all other units in that layer are inactive. By passing the activation from the Result layer multiplicatively with activation in the Value Input Layer through the gating neurons, only the Memory Layer units connected with the current choice are reinforced on any particular trial, all other units only decay. Activation in the Value Input Layer (See Figure 1) is calculated using the current win and/or current loss divided by the starting balance of $\$ 2,000$ or the maximum card seen so far. This gives a value between 0 and 1 , which, once gated (as discussed above), becomes the input into the Memory Layer, $\mathrm{y}_{\alpha}$ (See Equation 1) which is passed through a time-averaging equation. The timeaveraging equation (Equation 1) represents how information decays over time in the memory layer and how present information is valued against past knowledge. 


$$
\operatorname{act}(t)=\tau \cdot \operatorname{act}(t-1)+(1-\tau) \cdot y_{\alpha}(t)
$$

where $y_{d}(t)$ is the input activation from the Value Input Layer gated to the relevant Memory Layer unit at time $t$; and $\operatorname{act}(t)$ is the units activation at time $t$.

Finally, on each trial, the activation from the negative memory units is subtracted from the activation in the corresponding positive memory units and then passed through a sigmoidal function onto the connected Response Layer units. Once all Response Layer units have received their inputs from the memory layer, and additional random noise has been added, a winner-takes-all system ensures that the unit with the highest activation becomes the choice for the next trial. The addition of random noise allows for increased exploration, preventing action choice being completely greedy. Note also that, after each trial units in the Value Input Layer and the Response layer are set to zero. Whereas the Memory Layer units (which hold a measure of past experience related to a particular choice) and those units in the Result Layer, holding the Response Layer result from the current trial, to be used as the choice in the next trial, retain activation.

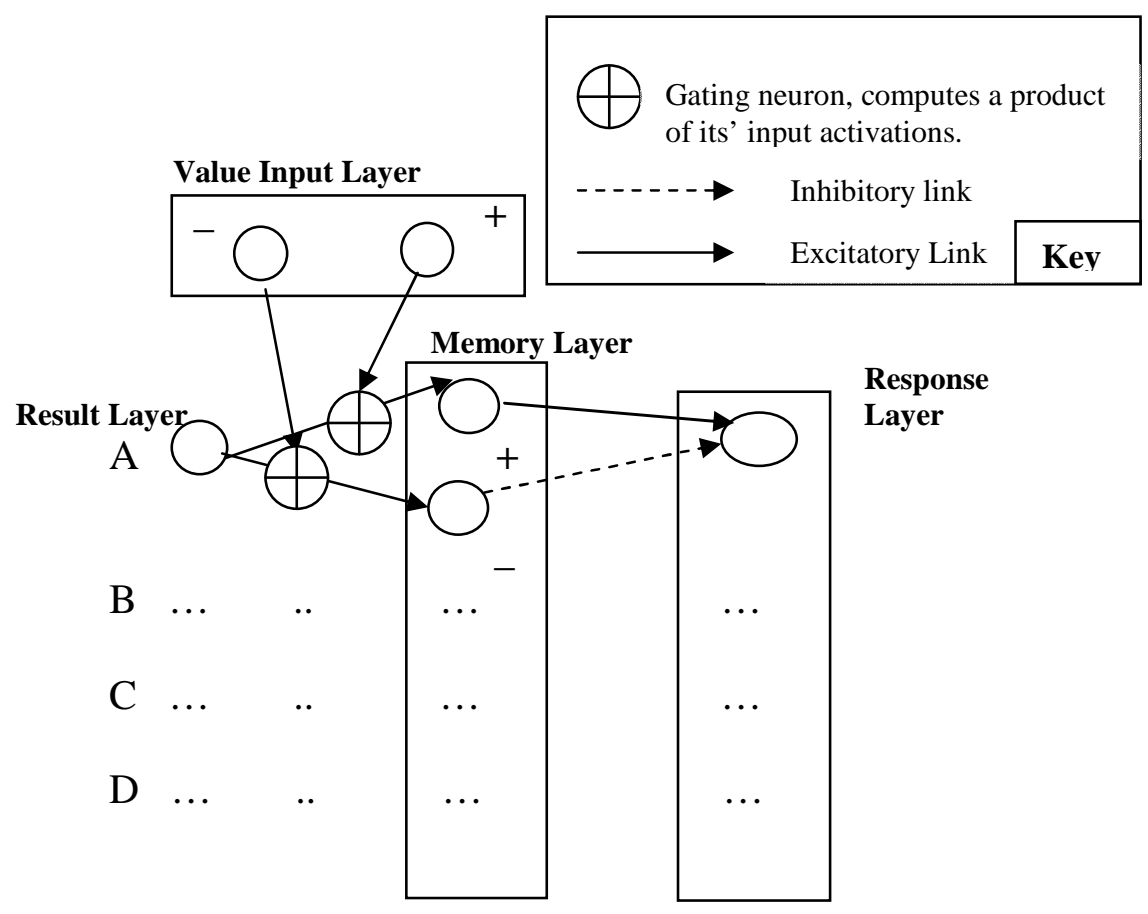

Figure 1. Diagram of Neural Network used for the Iowa Gambling Task. It shows the repetition of the basic architecture for each choice in the problem space. In the IGT there are four choices/decks. 


\section{Results}

In the exploration of the parameter space for $\tau$ (See equation 1), it was found that the normal control deck choice profiles could only be reproduced with a rising value of $\tau$ over the course of the IGT. However, the closest to the deck choice profiles for the VMF patients was best replicated using a constant $\tau=0.52$. (This gave an average variance in data points between the simulation and the real data of $2 \%$ ). The important factor in reproducing the normal controls data was not a particular growth function of $\tau$ across trials, but that it began at $\tau=0.52$ or below and rose to 0.9 by the 80th trial and then stayed constant at 0.9 for the remaining 20 trials. The 80 th trial was chosen as the limiting point as this is when many subjects, both patients and controls, gained conceptual knowledge of the advantageous and disadvantageous decks and therefore a conscious strategy (Bechara et al, 2000b). The essential quality was an increasing $\tau$ over time/trials: with logarithmic growth from 0-0.9 in 80 trials; sigmoidal growth from $0.3-0.9$ in 80 trials and linear growth from $0.52-0.9$ in 80 trials, each giving an average variance of approximately $6 \%$ between the real data and that from the simulation. Interestingly, the starting point for linear growth of $\tau$ for normal subjects is the same as the constant value most accurate in reproducing the VMF patient data.

\section{Conclusions}

The required growth in $\tau$ suggests that normal controls build up a knowledge of the task in hand by valuing new information more early in the task than later. This has the consequence that previous learning, as activation, is built up and not lost as rapidly as with a constant $\tau$ where new information can never have more than the initial 1- $\tau$ influence. Therefore, if $\tau$ is initially low and then increases over trials, new information has a greater impact early in the task. These simulation results are largely consistent with the myopia for future consequences theory of VMF patient behaviour. Although the model fleshes out the theory somewhat, by suggesting that what is missing in VMF patients is the capacity to adjust the trade-off between responding to new reward/punishment information and allowing past experience to affect the current choice. That is, in post-initial phases of the Iowa Gambling Task VMF patients are unable to switch to a strategy in which past experience is emphasized and preserved. By its nature such a strategy would focus more on future consequences, since learning that pays heed to past experience is more likely to enable decisionmaking that is more tuned to longer-term future consequences. This leads to the suggestion that the ventromedial prefrontal cortex is important in sustaining activation for goal directed behaviour by integrating relevant past experience. Perhaps VMF patients are unable to hold onto or access this information. It has 
been proposed that this retention of goal relevant information is a key role of the prefrontal cortex. "How does the PFC (Prefrontal Cortex) 'latch' onto goalrelevant information and maintain it without disruption? Several ideas have been suggested. These typically employ a form of gating signal that instructs the PFC network when to maintain a given activity state. This gating signal may come from dopaminergic (DA) neurons in the midbrain and basal ganglia." (p1370, Squire, 2003). This could be related to the growth in $\tau$ over trials.

Damasio (1994) has suggested that VMF patients' decision-making processes are no longer properly supported by 'somatic markers'. The authors of this paper only argue that VMF patients have a limited capacity in integrating relevant past information into their decision processes, without going as far as stating what form this information takes, e.g. affect or 'cold' cognitive. It is known that information is passed onto and analyzed in some way within consciousness as some VMF patients (50\% by 80 cards) report 'conceptual' knowledge of the best decks, $70 \%$ of controls reach this state by 80 cards (Bechara et al, 2000b). But unlike controls VMF patients do not reflect this knowledge in their subsequent behaviour and still pick more or less randomly. It seems that conceptual conclusions for these patients are insufficient for behavioural control. This could be similar to when some addicts 'know of what not to do', but lack the behavioural control to prevent repeated use. Do theories of dual systems, provide a potential solution, where the non-conscious System 1 (Evans, 2003) in VMF patients is making decisions containing affect, with little or no influence from conscious conclusions (System 2)? The current simulation is considered to be at a level of abstraction above that describing a dual systems theory, but it could be modified to become the basis of a representation of 'somatic-makers' in the System 1 part of a dual-process model, with the relative influence of System 2 over System 1 controlling the growth of $\tau$ across trials.

\section{References}

Bechara A., Damasio A.R., Damasio H. and Lee G.P. (1999). Different contributions of the human amygdala and ventromedial prefrontal cortex to decision-making. The Journal of Neuroscience, 19(13):5473-5481.

Bechara A., Tranel D. and Damasio H. (2000a). Characterization of the decision-making deficit of patients with ventromedial prefrontal cortex lesions, Brain 123: 2189-2202.

Bechara A., Tranel D. and Damasio H. (2000b). Emotion, Decision Making and the Orbitofrontal Cortex, Cerebral Cortex 10: 295-307.

Damasio A.R. (1994). Descartes Error: Emotion, Reason and the Human Brain. New York: Grosset/Putnam.

Evans J.St.B.T. (2003). In two minds: dual-process accounts of reasoning, Trends in Cognitive Sciences, Vol 7, No 10: 454-459.

Squire L.R. (2003). Fundamental Neuroscience. Amsterdam; London : Academic. Wagar B. and Thagard P. (2004). Spiking Phineas Gage: A Neurocomputational Theory of Cognitive-Affective Integration in Decision Making, Psychological Review, Vol 111, No 1: 67-79. 